\title{
O USO DA NEUROMODULAÇÃO NO TRATAMENTO DAS DISFUNÇÕES DE ELIMINAÇÕES
}

\author{
NEUROMODULATION IN THE TREATMENT OF ELIMINATIONS DYSFUNCTION \\ SYNDROME
}

\author{
Lisieux Eyer de Jesus, TCBC-RJ'1; Kathia Nery²
}

\begin{abstract}
RESUMO: Objetivo: Apresentar a experiência clínica com o uso de neuromodulação através de eletroestimulação transcutânea no tratamento das disfunções de eliminação. Métodos: Foram analisados retrospectivamente seis pacientes com disfunções de eliminação submetidos à neuromodulação através de eletroestimulação percutânea peri-sacral. Todos os pacientes incluídos eram refratários ao tratamento clínico convencional e a eletroestimulação foi utilizada como terapia de resgate. As sessões terapêuticas eram semanais e o seguimento mínimo foi de seis meses. Resultados: Todos os pacientes obtiveram melhora clínica. Quatro pacientes não necessitaram de mais medicação. Todos aceitaram bem o tratamento e referiram melhora da qualidade de vida. Conclusão: A eletroestimulação percutânea mostrou-se útil no tratamento das disfunções do assoalho pélvico e da musculatura vesical (Rev. Col. Bras. Cir. 2007; 34(6): 392-397).
\end{abstract}

Descritores: Transtornos urinários; Micção; Constipação intestinal; Estimulação Elétrica Transcutânea do Nervo.

\section{INTRODUÇÃO}

Os últimos 20 anos trouxeram grandes mudanças conceituais com relação aos distúrbios funcionais da musculatura pelvi-perineal. A correlação fisiopatológica entre os distúrbios vesicais e os da evacuação foi fundamental: o funcionamento integrado (neuro-muscular) do assoalho perineal representou uma explicação satisfatória para a associação freqüente entre constipação crônica e problemas urológicos, a seguir denominada síndrome da disfunção das eliminações Koff et al ${ }^{1}$.

Novas linhas de pesquisa sugeriram um papel fundamental, previamente ignorado, das informações neurais aferentes na coordenação dos tratos urinário e intestinal distais. O urotélio tem um papel integrativo, atuando como um sincício funcional com funções proprioceptivas.

Exames 'in vivo' com PET-scan demonstraram a existência de modulação funcional do aparelho urinário e assoalho pélvico em vários níveis do sistema nervoso central.

A alta frequência de efeitos colaterais sérios com drogas anticolinérgicas e a relativa ineficácia do tratamento com laxativos, técnicas funcionais e drogas moduladoras da atividade vesical determinaram a busca de novas táticas terapêuticas.

Apresentamos a nossa experiência clínica com o uso de eletroestimulação percutânea (EP) semanal perisacra no tratamento de uma coorte selecionada de portadores de distúrbios graves das funções de eliminação, previamente refratários ao tratamento clínico durante longos períodos.

\section{MÉTODOS}

Foram analisados os prontuários de seis pacientes cujas idades variavam entre 5 e 14 anos que apresentavam disfunções de eliminação e foram submetidos à eletroestimulação percutânea (EP) peri-sacra $(\mathrm{S} 2-\mathrm{S} 4)$ e torácica (T11 -L2). A EP foi aplicada uma vez por semana com duração de 40 minutos cada uma durante 10 semanas.

O tratamento foi ministrado por um cirurgião pediatra, responsável pela análise e seguimento clínico dos casos e um médico acupunturista, responsável pela aplicação da EE e análise clínica dos pacientes durante as sessões. Em todos os pacientes aqui apresentados a eletroestimulação foi utilizada como terapia de resgate. Foram excluídas da análise todas as crianças que apresentavam distúrbios neurológicos e megacólon agangliônico.

\section{RESULTADOS}

1. MYBV, masculino cinco anos. História pregressa de heminefrectomia polar superior direita para tratamento de duplicação complicada por ureterocele e exclusão funcional do rim direito. Apresentava disfunção miccional residual e infecções repetitivas do trato urinário, causadas pela conjunção de obstrução mecânica por 'flap' mucoso derivado da ureterocele ectópica e distúrbio miccional (micção infreqüente). Foi submetido à ressecção da ureterocele e feito reimplante ureteral direito (técnica de Politano), apresentando retenção urinária e fecal refratárias aos fármacos e à re-educação

1. Cirurgiã Pediátrica do Hospital Universitário Antônio Pedro, Universidade Federal Fluminense, Niterói - RJ e Hospital dos Servidores do Estado, Rio de Janeiro- RJ

2. Médica Acupuntora; Professora do Serviço de Acupuntura Médica Contemporânea da Universidade Federal Fluminense, Niterói, RJ.

Recebido em 25/04/2007

Aceito para publicação em 27/06/2007

Conflito de interesses: nenhum

Fonte de financiamento: nenhuma

Trabalho realizado Hospital Universitário Antônio Pedro, Universidade Federal Fluminense, Niterói - RJ. 
miccional no pós-operatório. Persistiu dependente de micção por cistostomia e de evacuação com ajuda de clister durante oito semanas.

A cistografia era normal, não evidenciando obstrução da saída vesical. Não foi encontrada lesão cirúrgica da inervação vesical, (reimplante unilateral, sem manipulação retro-vesical mediana ou à esquerda, intervenção trigonal limitada à metade direita do trígono). $\mathrm{O}$ paciente foi então submetido à eletroestimulação, sem o uso de medicamentos auxiliares. Era aplicado clister evacuativo em dias alternados. A constipação intestinal se resolveu após duas semanas de tratamento e a micção voluntária foi restabelecida após três semanas, retirando-se a cistostomia após quatro semanas. A eletroestimulação semanal foi mantida durante quatro meses. O paciente persistiu evacuando espontaneamente uma vez/dia, com micção voluntária de padrão normal (controle urodinâmico e ultrasonográfico) sem o uso de tratamentos auxiliares, após três anos de seguimento. Apresenta episódios raros de incontinência do riso (< um episódio a cada dois meses), atribuídos à disfunção esfinctérica associada às ureteroceles ectópicas. Houve plena aceitação do tratamento pela criança e familiares, que afirmam que repetiriam o tratamento e o recomendariam a outros pacientes.

2. BVGC, masculino, seis anos. Portador de infecções urinárias de repetição desde um ano de idade. Hidronefrose bilateral grave, refluxo vésico-ureteral grave bilateral com várias cicatrizes renais, creatinina elevada (mensuração imediatamente anterior ao tratamento $1,8 \mathrm{mg} / \mathrm{dL}$ ). O exame urodinâmico demonstrava ausência de sensibilidade vesical à distensão, capacidade vesical muito aumentada, detrusor inativo, micção voluntária ausente, possível sob demanda através de manobra de Valsalva (Figura 1). Constipação crônica refratária, evacuando exclusivamente através de clister. Sem melhora clínica apesar do uso de cateterismo intermitente limpo e clisteres evacuativos diários, mantendo $>4$ infecções urinárias/ano, piora clínica progressiva, déficit estaturo-ponderal, prejuízo grave da atividade escolar. Encaminhado para derivação urinária incontinente. Quatro semanas após o início da eletroestimulação passou a evacuar espontaneamente 1-2 vezes/dia sem o uso de laxativos. Após dois meses passou a executar micção programada a cada $4 \mathrm{~h}$, seguida de cateterismo intermitente limpo (Figura 2). Foram observados resíduos pós-miccionais progressivamente menores até se registrar resíduos $<15 \%$ do volume vesical, quando prescindimos do cateterismo intermitente. O paciente demonstrou grande aumento da diurese/dia, sugerindo fenômeno de diurese pós-obstrutiva. Persiste sob controle ultrasonográfico e urodinâmico, exibindo resíduos pós-miccionais normais e sem apresentar infecções de trato urinário há mais de um ano. Houve melhora progressiva da hidronefrose, da retomada do crescimento. O desenvolvimento da criança e da atividade escolar. Seguimento de dois a oito meses. Houve plena aceitação do tratamento pela criança e familiares, que são extremamente gratos e referem à eletroestimulação como única forma de tratamento que foi capaz de melhorar o quadro clínico. Afirmam que repetiriam o tratamento e o recomendariam a outros pacientes.
3. JWSC, masculino 5 anos. Portador de distúrbio de comportamento e problemas sócio-familiares graves. Retenção urinária e fecal crônicas, escape fecal constante, evacuação exclusivamente mediante clisteres. Micção por transbordamento, globo vesical sempre palpável. Dependente de cateterismo intermitente para esvaziamento vesical, recusado pela criança. Episódios recorrentes de orquiepididimite. Urodinamicamente detrusor hipoativo, bexiga com capacidade muito aumentada, ausência de sensibilidade ao enchimento, ausência de micção voluntária (Figura 3). Seis semanas após o início da eletroestimulação passou a ter micção voluntária sem o auxílio de drogas ou cateterismo, que se mantém após dois anos de seguimento. O controle ultra-sonográfico demonstra resíduos pósmiccionais. A uretrocistografia retrógrada normais. A função evacuativa não demonstrou melhora satisfatória: embora exiba eventuais episódios de evacuação voluntária continua usando clisteres em dias alternados para esvaziamento colônico, mantendo bom controle da constipação e ausência de escape fecal. Houve plena aceitação do tratamento pela criança e familiares, que, no entanto, demonstraram disciplina inadequada para freqüentar as sessões em longo prazo, por problemas sociais, a partir da obtenção da normalidade miccional.

4. MF, feminina 9 anos. História de correção perinatal de fístula reto-vestibular (anoretoplastia sagital posterior perinatal, sem colostomia de proteção) com complicação imediata que exigiu reintervenção. Seguiram-se constipação crônica e mega-reto adquirido refratários a várias formas de trata-
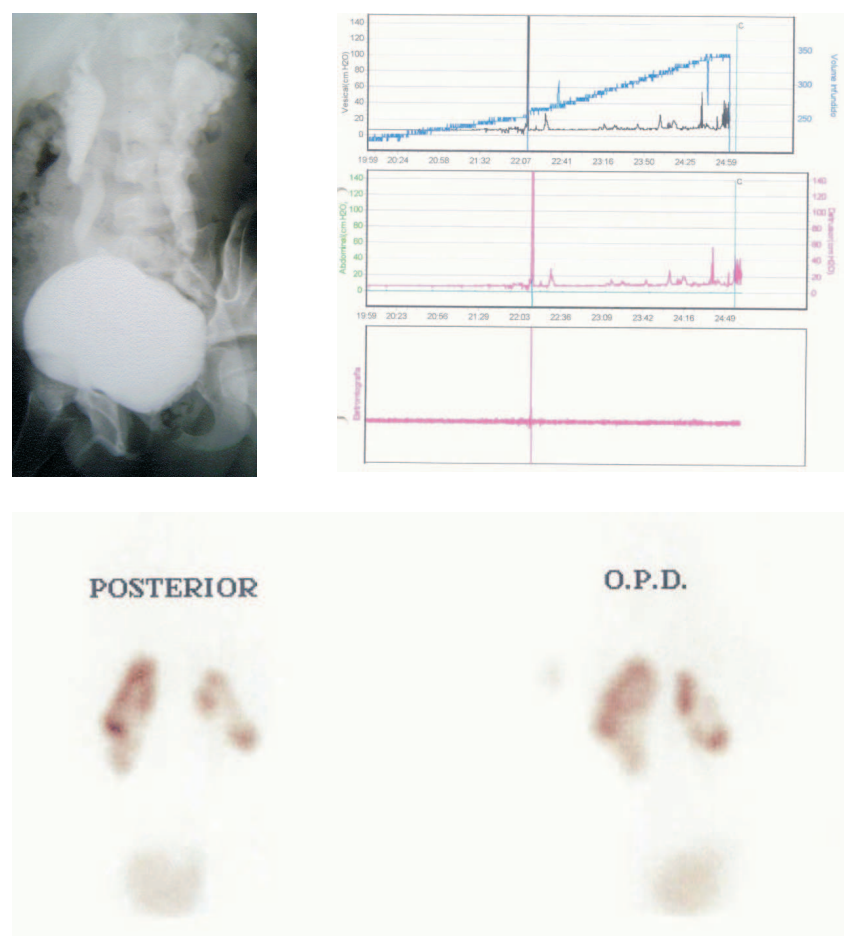

O.P.D.

Figura 1 - Paciente 2, uretrocistografia miccional, estudo urodinâmico e cintigrafia renal estática pré-tratamento, demonstrando refluxo vésico-ureteral grave bilateral, detrusor inativo e lesão renal bilateral grave. 


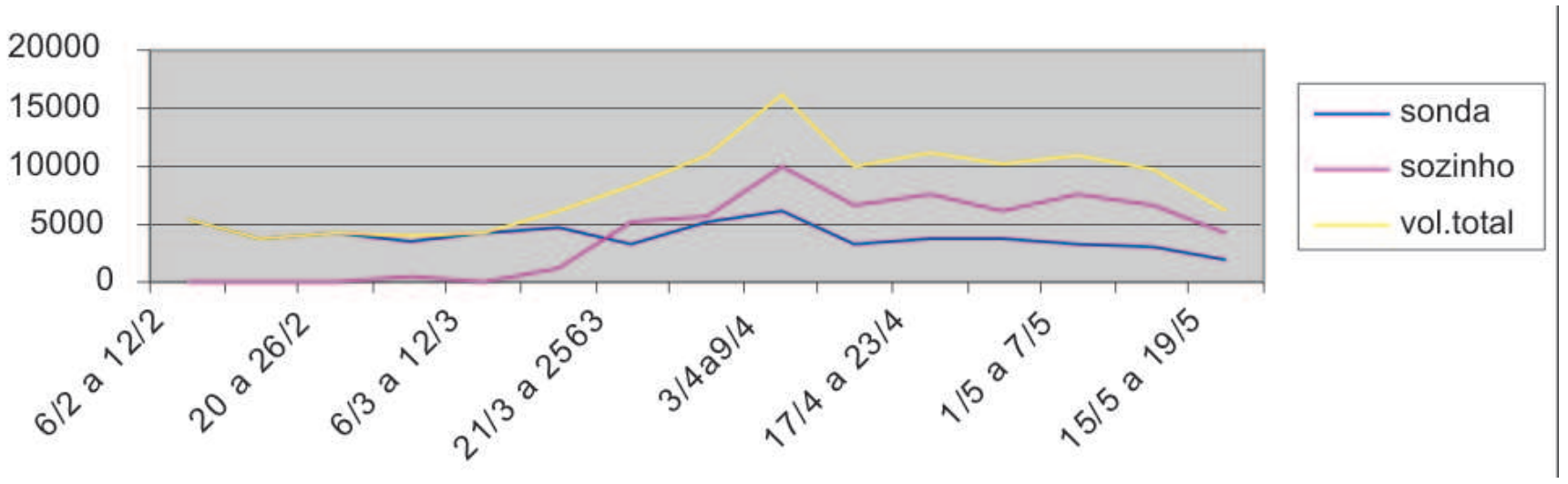

Figura 2 - gráfico demonstra a progressão da micção voluntária e diurese total após o início da eletroestimulação.

mento (Figura 4), sem queixas urinárias. Megacólon agangliônico e estenose anal pós-operatórios foram excluídos. Várias internações hospitalares para desimpacção fecal e escape fecal constante a levaram à exclusão escolar. Após desimpacção fecal foi prescrita terapia com polietilenoglicol oral em dias alternados, com melhora da constipação, mas escape fecal constante que obrigava a paciente a usar fraldas. Episódios de urgência evacuativa eram freqüentes, acompanhados de incontinência. Após seis semanas da eletroestimulação evacuando diariamente, em uso de polietilenoglicol oral apenas duas vezes/ semana. Escape fecal raro (< um episódio/semana), melhora da propriocepção anoretal, com controle voluntário eficaz dos episódios de urgência evacuativa, e resolução da incontinência. Não usa fraldas/ protetores íntimos atualmente, retornou às atividades escolares. Houve plena aceitação do tratamento pela criança e familiares, que afirmam que esta foi a única instância de tratamento satisfatório recebido pela criança e repetiriam a terapia ou a recomendariam a outros pacientes. Seguimento clínico de seis meses.

5. JP, masculino 7 anos. Portador de enurese diária e hiperatividade vesical (síndrome de urgeincontinência e micção incoordenada, demonstrada em estudo urodinâmico). Uso inadequado de anticolinérgico e recusa terapêutica por efeitos colaterais neurológicos centrais da droga (oxibutinina). Uso irregular da eletroestimulação por problemas familiares. Dois meses após o início da terapia apresentando 4-5 noites secas/semana, freqüência miccional menor e resolução da urgencontinência. A melhora se mantém apesar da interrupção da eletroestimulação há cerca de três meses (seguimento clínico de nove meses). Houve plena aceitação do tratamento pela criança e familiares. $\mathrm{O}$ paciente freqüentemente dorme durante as sessões.

6. JQ, masculino 14 anos. Portador de problemas sócio-familiares graves, distúrbio de comportamento e oligofrenia moderada. Constipação crônica refratária, múltiplas internações hospitalares para desimpacção fecal, clisteres evacuativos pouco eficientes, megaretosigmóide (Figura 4). Escape fecal constante, rejeitado no ambiente escolar e familiar, abandonado pelos pais, adotado por avó paterna. Polaciúria e micções repetitivas de baixo volume, quadro fortemente sugestivo de hiperatividade vesical. Não foi possível obter estu- do urodinâmico. Seis semanas após o início da eletroestimulação paciente passou a evacuar diariamente sem usar drogas ou clisteres e se mantém com evacuações diárias após seis meses de interrupção da terapia (seguimento clínico de nove meses), embora evacue eventualmente em local inapropriado devido ao distúrbio psiquiátrico. Não foi possível avaliar plenamente a evolução do distúrbio miccional, embora o responsável refira melhora da polaciúria. Houve plena aceitação do tratamento pela criança (apesar do distúrbio psiquiátrico) e familiares, que afirmam que esta foi a única forma
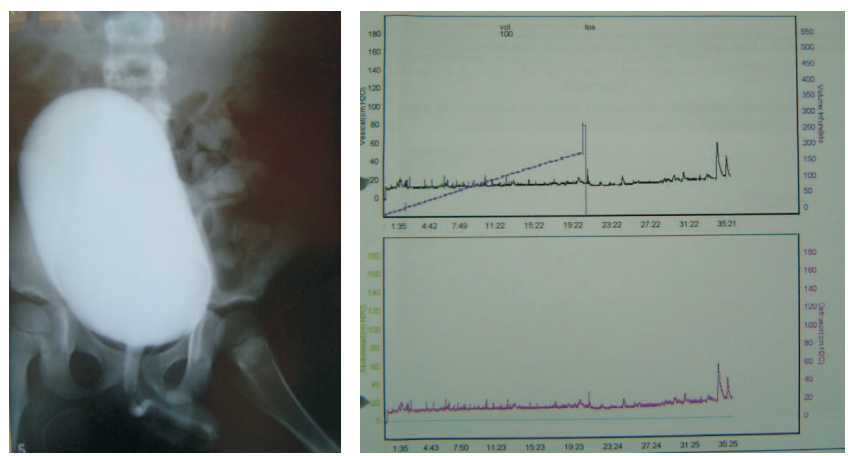

Figura 3 - Caso clínico número 3. Uretrocistografia miccional e urodinâmica pré-tratamento, demonstrando bexiga regular com tamanho muito aumentado e detrusor inativo.
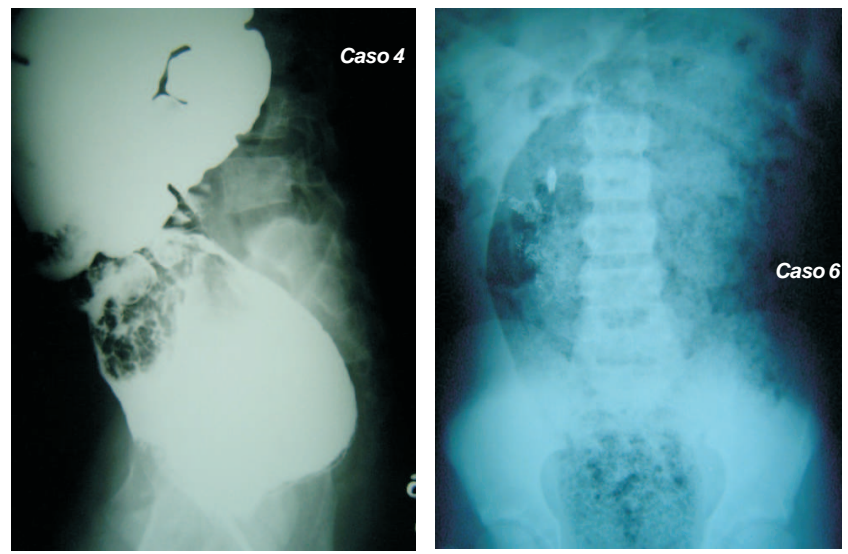

Figura 4 - Acentuada constipação crônica refratária. Radiografias mostrando a gravidade da dilatação intestinal e retenção fecal envolvidos (Pacientes MF e JQ). 
de terapia capaz de exibir resultados. Repetiriam o tratamento em caso de necessidade ou o recomendariam a outros pacientes.

\section{DISCUSSÃO}

A incoordenação entre os sistemas de eliminação (detrusor e peristalse retal) e de controle de eliminação (esfincteres urinários e anal) em crianças sem doença neurológica envolve anomalias da propriocepção vesical/ retal/ perineal e/ou dos mecanismos integrativos (epitelial-parietalneuromuscular, aferente-eferente). Embora presumivelmente adquiridas estas síndromes não costumam ter distúrbios comportamentais como causa. É muito mais freqüente que as alterações de comportamento sejam conseqüência dos problemas clínicos (principalmente incontinência), da necessidade de intervenções médicas repetitivas e das tentativas de compensação social pelo paciente e familiares. Apesar da correlação estatística ser clássica, a associação entre problemas clínicos da evacuação e distúrbios miccionais foi valorizada no final dos anos 901. Há uma nova forma de avaliar estes distúrbios comuns na prática clínica, que correlaciona funcionalmente constipação crônica e síndromes urológicas de hiperatividade vesical ou retenção urinária. A assunção de uma unidade neuro-funcional integrada pelvi-perineal coordenando simultaneamente as atividades vésico-uretrais e retoanais, é uma idéia relativamente nova e atraente, explica logicamente os sinais e sintomas e permite uma abordagem terapêutica única para todas as expressões clínicas de uma síndrome perineal neuro-muscular disfunção de eliminações ${ }^{2}$.

Em termos fisiológicos, a valorização de mecanismos aferentes no controle da atividade retal/ vesical infere que a propriocepção é essencial, atuando através de arcos reflexos locais e centrais ou interações neuro-neurais periféricas. Um exemplo deste último mecanismo é a interação entre "inputs" aferentes pelo nervo pudendo e nervos autônomicos no plexo hipogástrico, que possivelmente é responsável pelos efeitos terapêuticos dos eletroestimuladores protéticos do nervo pudendo no tratamento da bexiga hiperativa. O reconhecimento do urotélio como um sincício funcional sinalizador neural local sugere a existência de um sistema proprioceptivo coordenador nos dois aparelhos.

A terapêutica convencional envolve modulação farmacológica da atividade vesico-esfincteriana (com anticolinérgicos e alfa-bloqueadores), esvaziamento vesical programado (cateterismo intermitente limpo, micção programada e técnicas de treinamento urológico - biofeedback) e limpeza intestinal através de laxativos e enemas. Infelizmente todas estas formas de tratamento têm sido questionadas ${ }^{3}$. A aceitação do cateterismo intermitente limpo e de enemas pelos pacientes neurologicamente saudáveis é precária, e é freqüente a recusa ou o abandono do tratamento. Estes procedimentos também não são bem aceitos pelos familiares. Em casos mais graves há indicação de desvios urinários incontinentes (vesicostomia), condutos cateterizáveis extra-anatômicos do tipo Mitrofanoff e Malone.

A eficácia e a gravidade dos para-efeitos das drogas anti-colinérgicas têm sido discutidas recentemente. Uma metanálise ${ }^{4}$ demonstrou que o efeito dos anticolinérgicos é bastante discutível frente aos placebos (efeito comparativo apenas 1,4 vezes melhor). Informações recentes em adultos atentam para defeitos intelectivos pelo uso de anticolinérgicos (o efeito prejudicial da oxibutinina nos mecanismos de memória corresponderia em média a 10 anos de envelhecimento $)^{5} \mathrm{e}$ síndromes demenciais Alzheimer-like, reversíveis com a retirada das drogas ${ }^{6}$. Não há estudos de boa qualidade técnica analisando os efeitos destas drogas sobre o cérebro humano infantil em processo de maturação.

Os dados disponíveis com relação aos alfabloqueadores (presumivelmente capazes de deprimir simultaneamente a atividade esfinctérica e do detrusor) são escassos, especialmente em pacientes não neurológicos ${ }^{7}$.

Com relação aos programas de biofeedback, embora seguros, têm sua eficiência muito discutida, variando entre extremos de autor para autor, assim como a durabilidade dos efeitos favoráveis em longo prazo. Exigem um esforço logístico grande da equipe de saúde, da criança e dos familiares, tratamentos longos, uso de tecnologia informatizada e maturidade do paciente. Seu uso se limita seu uso a crianças mais velhas, capazes de se concentrar, compreender e executar as instruções necessárias.

As propostas de estimulação neural para modular a atividade do trato urinário baixo e do esfíncter anal são recentes em literatura para o tratamento de incontinências urinárias e fecais, em sua maioria reservadas a pacientes sem doença neurológica de base. A forma mais conhecida é a eletroestimulação direta de da terceira raiz sacra através da inserção percutânea de um eletrodo através de aparelho inserido no subcutâneo: os resultados relatados são bastante satisfatórios ${ }^{8-9}$, porém trata-se de procedimento invasivo, caro e de difícil acesso. Outros autores vêm propondo o uso de aparelho protético estimulador do nervo pudendo.

A neuromodulação exerce um efeito de recondicionamento fisiológico, possibilitando a remodelação de sinapses através dos mecanismos de neuroplasticidade e permitindo, teoricamente, o recondicionamento neural definitivo $^{10}$. Artigos utilizando várias metodologias de eletroestimulação periférica (peri-tibial, suprapúbica, perisacral, perineal e outras) foram descritos, com melhora ou cura em várias coortes de adultos com disfunção miccional (período médio de tratamento de 12 semanas, uma vez/semana $)^{11-13}$. Alguns autores usam acupuntura clássica, também com bons resultados ${ }^{14-15}$. Todos os autores sugerem que a melhora se deve à modulação neural e aperfeiçoamento dos mecanismos integrativos, agindo principalmente sobre descargas aferentes.

A extensão direta destes resultados para crianças é questionável, considerando a fisiopatologia diferente dos distúrbios funcionais nas diversas idades e nuances de maturação, motivação e comportamento. Há poucos relatos de eletroestimulação periférica no tratamento de distúrbios miccionais pediátricos. Hoebecke et al. (estimulação peri-tibial percutânea semanal com agulha, 20 sessões) demonstram melhora em 17/28 de bexigas hiperativas e 16/19 de retenção urinária não neuropática. Houve $61 \%$ de melhora nos sintomas de urgência, 69\% diminuição perdas involuntárias, $84 \%$ normalização na freqüência miccional e $43 \%$ melhora na 
urofluxometria $^{16}$. Há relatos de resultados favoráveis em crianças portadoras de urge-incontinência, bexiga hiperativa e em 5/7 pacientes com retenção urinária. Os resultados foram desfavoráveis para bexigas neurogênicas ${ }^{17}$. Usando estimulação domiciliar Bower et al. obtiveram $73 \%$ de melhora em crianças portadoras de urge-incontinência ${ }^{18}$. Embora mais que 1/3 das crianças portadoras de distúrbio miccional apresentem alguma forma de atividade inapropriada do assoalho pélvico, casos com dissinergia do assoalho pélvico não são relatados separadamente e as coortes apresentadas não são uniformes, têm amostragens pequenas e sem grupo-controle. Embora concordem quanto aos níveis de segurança e evidências iniciais de eficácia, todos os autores sugerem estudos controlados para a demonstração indiscutível da utilidade das técnicas ${ }^{19}$. Considerando os argumentos éticos envolvidos, em todos estes estudos as técnicas de estimulação foram usadas em associação ao tratamento convencional (farmacológico), o que dificulta a avaliação isolada dos resultados das técnicas de eletroestimulação, mesmo considerando pacientes refratários ao tratamento farmacológico após mais de dois anos de uso.

Em julho de 2006 foi publicado pelo Grupo de Urologia da UNIFESP/SP/Brasil ${ }^{20}$ um estudo-piloto prospectivo, randomizado e com um padrão razoável de uniformização, certamente um marco. Os autores apresentam uma coorte de 36 crianças entre 3 e 14 anos de idade, neurologica e anatomicamente sãs, todas portadoras de hiperatividade vesical (urge-incontinência e infecção urinária de repetição), distribuidas de forma randomizada num grupo tratado através de eletroestimulação perisacra percutânea $(\mathrm{G} 1, \mathrm{n}=19)$ e outro $(\mathrm{G} 2, \mathrm{n}=17)$ tratado através de técnicas de biofeedback. Em nenhum paciente foram usadas drogas moduladoras da atividade vesical. 12/19 pacientes no Grupo 1 e 10/17 no Grupo 2 foram consideradas curadas. No G1 mais seis pacientes tiveram $>80 \%$ de melhora clínica e não houve nenhum paciente que não exibiu melhora. No G2 cinco pacientes só apresentaram $<50 \%$ e seis casos não apresentaram qualquer melhora. Adicionalmente, os pacientes do G2 sem melhora ou com recidiva de sintomas foram eficientemente resgatados através de eletroestimulação. No G1 aproximadamente $40 \%$ dos portadores de enurese persistiram assintomáticos e mais $30 \%$ melhoraram após o tratamento. Todos os portadores de constipação neste grupo melhoraram (6/13) ou ficaram curados do sintoma $(5 / 13)$.

No estudo que ora apresentamos as características são diversas dos demais: os pacientes não foram selecionados a partir do diagnóstico causal da disfunção, agrupando várias causas dos sintomas finais de incontinência e síndromes retentivas e são todos casos graves refratários à terapia convencional. Em todos os casos a eletroestimulação foi utilizada como terapia de resgate, com bons resultados. São necessários mais estudos com grupos maiores e metodologias uniformes, preferencialmente de natureza multicêntrica, para determinar a técnica e periodicidade ideais para eletroestimulação em crianças, os limites de eficácia terapêutica e as indicações, de forma comparativa a outros métodos, considerando em especial a baixíssima morbidade, logística relativamente simples, baixo custo e excelente aceitação pelos pacientes.

\begin{abstract}
Objective: To present a clinical trial with neuromodulation through percutaneous electrical stimulation in the treatment of elimination dysfunctions. Methods: Six patients with dysfunctional elimination diagnosis were included and their data were analyzed retrospectively, regarding percutaneous sacral electrical stimulation. All the patients included, have had unsuccessful conventional treatments and the electrical stimulation was used as rescue therapy. All the sessions were applied in weekly fashion for six months. Results: All the patients have had a positive clinical outcome, with four of them interrupting their medication. All the patients accepted well the therapy and referred an improvement in their quality of life. Conclusion: The electrical percutaneous stimulation, showed to be effective on vesical muscle and pelvic floor dysfunctions.
\end{abstract}

Key words: Urinary dysfunctions, urination, intestinal constipation, electrical nerve stimulation.

\section{REFERÊNCIAS}

1. Koff SA, Wagner TT, Jayanthi VR. The relationship among dysfunctional elimination syndromes, primary vesicoureteral reflux and urinary tract infections in children. J Urol. 1998; 160 (3 pt 2):1019-22.

2. Reitz A, Wefer B, Schurch B. New understanding of central and peripheral interaction between bladder and sphincter function. EAU Update Series. 2004; 2(4):153-60.

3. Sureshkumar P, Bower W, Craig JC, Knight JF. Treatment of daytime urinary incontinence in children: a systematic review of randomized controlled trials. J Urol. 2003; 170(1):196-200; discussion 200.

4. Herbison P, Hay-Smith J, Ellis G, Moore K. Effectiveness of anticholinergic drugs compared with placebo in the treatment of overactive bladder: systematic review. BMJ. 2003; 326(7394):841-4.

5. Kay G, Crook T, Rekeda L, Lima R, Ebinger U, Arquinzoniz M, Steel M. Differential effects of the Antimuscarinic Agents Darifenacin and Oxybutynin ER on the Memory in Older Subjects. Eur Urol. 2006; 50(2):317-26. Epub 2006 Apr 19.

6. Ancelin ML, Artero S, Portet F, Dupuy AM, Touchon J, Ritchie K. Non-degenerative mild cognitive impairment in elderly people and use of anticholinergic drugs: longitudinal cohort study. BMJ. 2006; 332(7539):455-9.

7. Chapple C. Alpha antagonists - from initial concept to routine clinical practice. Eur Urol. 2006; 50(4):635-42. Epub 2006 Jul 26.

8. Abrams P, Blaivas JG, Fowler CJ, Fourcroys JL, Macdiarmid SA, Siegel SW, Van Kerrebroeck P. The role of neuromodulation 
in the management of urinary urge incontinence. BJU Int. 2003; 91(3):355-9.

9. Braun PM, Seif C, van der Horst C, Jünemann KP. Neuromodulation- sacral, peripheral and central: current status, indications, results and new developments. EAU Update Series. 2004; 2(4):187-94.

10. Carneiro NM. Fundamentos da acupuntura médica. Florianópolis: Sistema; 2001.

11. Vandoninck V, van Balken MR, Finazzi Agro E, Heesackkers JP, Debruyne FM, Kiemeney LA, Bemelmans BL. Posterior tibial nerve stimulation in the treatment of voiding dysfunction: urodynamic data. Neurourol Urodyn. 2004; 23(3):246-51.

12. van Balken MR, Vandoninck V, Gisolf KW, Vergunst H, Kiemeney LA, Debruyne FM, Bemelmans BL. Posterior tibial nerve stimulation as neuromodulative treatment of lower urinary tract dysfunction. J Urol. 2001; 166(3):914-8.

13. Govier FE, Litwiller S, Nitti V, Kreder KJ, Rosenblatt P. Percutaneous afferent neuromodulation for the refractory overactive bladder: results of a multicenter study. J Urol. 2001; 165(4):1193-8.

14. Chang PL. Urodynamic studies in acupuncture for women with frequency, urgency and dysuria. J Urol. 1988; 140(3):563-6.

15. Philp T, Shah PJ, Worth PH. Acupuncture in the treatment of bladder instability. Br J Urol. 1988; 61(6):490-3.

16. Hoebeke P, Renson C, Petillon L, Walle JV, de Paepe H. Percutaneous electrical nerve stimulation in children whit therapy resistant nonneuropathic bladder sphincter dysfunction: a pilot study. J Urol. 2002; 168(6):2605-8.
17. de Gennaro M, Capitanucci ML, Mastracci P, Silveri M, Gatti C, Monsiello G. Percutaneous tibial nerve neuromodulation is well tolerated in children and effective for treating refractory vesical dysfunction. J Urol. 2004; 171(5):1911-3.

18. Bower WF, Moore KH, Adams RD. A pilot study of the home application of transcutaneous neuromodulation in children with urgency or urge incontinence. J Urol. 2001; 166(6):2420-2.

19. Bower WF, Yeung CK. A review of non-invasive neuromodulation as an intervention for non-neurogenic bladder dysfunction in children. Neurourol Urodyn. 2004; 23(1):63-7.

20. Barroso U, Lordêlo P, Lopes AA, Andrade J, Macedo A, Ortiz V. Nonpharmacological treatment of lower urinary tract dysfunction using biofeedback and transcutaneous electrical stimulation: a pilot study. BJU Int. 2006; 98(1):166-71.

Como citar este artigo:

Jesus LE, Nery K. O uso da neuromodulação no tratamento das disfunções de eliminações: estudo clínico. Rev Col Bras Cir. 2007; 34(4). Disponível em URL: www.scielo.br/rcbc

Endereço para correspondência:

Lisieux Eyer de Jesus

R. Presidente Domiciano, 52/801

Boa Viagem

CEP 24210-270 - Niterói - RJ.

Tels.: (21) 2622-3843; (21) 9985-9737

E-mail: lisieux@uol.com.br 